IJMMS 25:8 (2001) 497-507

PII. S0161171201005312

http://ijmms.hindawi.com

(c) Hindawi Publishing Corp.

\title{
FIXED POINTS VIA A GENERALIZED LOCAL COMMUTATIVITY
}

\author{
GERALD F. JUNGCK
}

(Received 24 May 2000)

\begin{abstract}
Let $g: X \rightarrow X$. The concept of a semigroup of maps which is "nearly commutative at $g$ " is introduced. We thereby obtain new fixed point theorems for functions with bounded orbit(s) which generalize a recent theorem by Huang and Hong, and results by Jachymski, Jungck, Ohta, and Nikaido, Rhoades and Watson, and others.
\end{abstract}

2000 Mathematics Subject Classification. Primary 47H10, 54H25.

1. Introduction. By a semi-group of maps we mean a family $H$ of self maps of a set $X$ which is closed with respect to composition of maps $(f \circ g=f g)$ and includes the identity map $i_{d}(x)=x$, for $x \in X$. We often associate with a function $g: X \rightarrow X$ following semi-groups:

$$
O_{g}=\left\{g^{n} \mid n \in \mathbb{N} \cup\{0\}\right\},
$$

where $\mathbb{N}$ is the set of positive integers and $g^{0}=i_{d}$, and

$$
C_{g}=\{f: X \longrightarrow X \mid f g=g f\}
$$

A quick check confirms that $C_{g}$ is a semi-group.

If $H$ is a semi-group of self maps of a set $X$ and $a \in X, H(a)=\{h(a) \mid a \in H\}$. In particular, if $H=O_{g}, O_{g}(a)=\left\{g^{n}(a) \mid n \in \mathbb{N} \cup\{0\}\right\}$ and is called the orbit of $g$ at $a$.

In general, Lemma 3.2 and some theorems in Section 3 will be stated in the context of semi-metric spaces. A semi-metric on a set $X$ is a function $d: X \times X \rightarrow[0, \infty)$ such that $d(x, y)=d(y, x)$ for $x, y \in X$ and $d(x, y)=0$ if and only if $x=y$. A semi-metric space is a pair $(X ; d)$, where $X$ is a topological space and $d$ is a semi-metric on $X$. The topology $t(d)$ on $X$ is generated by the sets $S(p, \epsilon)=\{x \mid d(x, p)<\epsilon\}$ with the requirement that $p$ is an interior point of $S(p, \epsilon)$. A sequence $\left\{x_{n}\right\}$ in $X$ converges in $t(d)$ to $p \in X$ (denoted as $x_{n} \rightarrow p$ ) if and only if $d\left(x_{n}, p\right) \rightarrow 0$. We let $t(d)$ be $T_{2}$ (Hausdorff) to ensure unique limits. Thus, a metric space $(X, d)$ is a semi-metric space having the triangle inequality. For further details on semi-metric spaces, see, for example, [1, 4, 6].

If $g: X \rightarrow X$, a semi-metric space ( $X$; $d$ ) is complete ( $g$-orbitally complete) if and only if every Cauchy sequence (in the usual sense) in $X\left(O_{g}(x)\right)$ converges to a point of $X$. $g$ is continuous at $p \in X$ if and only if whenever $\left\{x_{n}\right\}$ is a sequence in $X$ and $x_{n} \rightarrow p$, then $f\left(x_{n}\right) \rightarrow f(p)$. And if $S$ is a bounded subset of $X, \delta(S)=\sup \{d(x, y) \mid x, y \in S\}$.

We are now ready to focus on the intent of this paper, namely, to introduce a generalized "local commutativity" and to demonstrate the concept's usefulness. 
2. Nearly commutative semi-groups. In [2], a semi-group $H$ of maps is said to be near-commutative if and only if for each pair $f, g \in H$, there exists $h \in H$ such that $f g=g h$. We generalize as follows.

DEFINITION 2.1. A semi-group $H$ of self maps of a set $X$ is nearly commutative (n.c.) at $g: X \rightarrow X$ if and only if $(f \in H)$ implies that there exists $h \in H$ such that $f g=g h$.

Of course, $O_{g}$ and $C_{g}$ are n.c. at $g$. Observe also that a near-commutative semigroup $H$ of self maps of a set $X$ is n.c. at each $g \in H$. The following provides for each $a \in(0, \infty)$ an example of a semi-group $H=S_{a}$ of self maps which is not nearcommutative but is n.c. at a particular $g: X \rightarrow X$.

EXAMPLE 2.2. Let $X=[0, \infty)$ and $a \in(0, \infty)$. Let $g(x)=a x$ and define

$$
S_{a}=\left\{a^{m} x^{n} \mid x \in[0, \infty), n \in \mathbb{N}, m \in \mathbb{N} \cup\{0\}\right\},
$$

where $S_{a}$ is nearly commutative (n.c.) at $g$. For if $f(x)=a^{m} x^{n}$ is a representative element of $S_{a}$, then $f g(x)=f(g(x))=a^{m}(a x)^{n}=a^{m+n} x^{n}$. We want $h(x)=a^{r} x^{s} \in$ $S_{a}$ such that $f g=g h$. Now, $g(h(x))=a\left(a^{r} x^{s}\right)=a^{r+1} x^{s}$, so we can let $s=n$ and $r+1=m+n$; that is, $r=m+(n-1)$. Since $n \in \mathbb{N}$ and $(n-1), m \in \mathbb{N} \cup\{0\}, s$ and $r$ so designated imply $h \in S_{a}$. Thus, $\left(f \in H=S_{a}\right)$ implies that there exists $h \in H$ such that $f g=g h$. Since $i_{d} \in S_{a}, S_{a}$ is clearly a semi-group, and we are finished. On the other hand, $S_{a}$ is not a near-commutative semi-group. For example, let $f(x)=a^{2} x^{2}$ and $h(x)=a^{2} x^{3}$. We want $t(x)=a^{r} x^{s}$ such that $f h=h t$. So we must have $3 s=6$ and $(2+3 r)=6$. But then $r=4 / 3$, and $r \notin \mathbb{N} \cup\{0\}$.

Now, let $\mathcal{M}_{n}$ and $\mathcal{N}_{n}$ denote the set of all $n \times n$ real matrices and the set of all nonsingular $n \times n$ real matrices, respectively. Then, both sets $\mathcal{M}_{n}$ and $\mathcal{N}_{n}$ are semigroups of linear transformations $A: \mathbb{R}^{n} \rightarrow \mathbb{R}^{n}$ relative to composition of maps (matrix multiplication).

EXAMPLE 2.3. $\mathcal{N}_{n}$ is n.c. For if $A, B \in \mathcal{N}_{n}$, there exists $C=B^{-1}(A B) \in \mathcal{N}_{n}$ such that $A B=B C$.

EXAMPLE 2.4. $\mu_{n}$ is n.c. at any $B \in \mathcal{N}_{n}$, by Example 2.3. But $\mu_{n}$ is not near commutative. For instance, if $n=2, B=\left[\begin{array}{ll}1 & 1 \\ 0 & 0\end{array}\right]$, and $A=\left[\begin{array}{ll}1 & 2 \\ 3 & 4\end{array}\right]$, there exists no $2 \times 2$ matrix $C$ such that $A B=B C$.

Now, let $g: X \rightarrow X$. Since any semi-group of self maps which commute with $g$ is a subset of $C_{g}$, we might hope that $H_{g}=\{f: X \rightarrow X \mid f g=g h$ for some $h: X \rightarrow X\}$ would be a maximal semi-group which is n.c. at $g$. However, $H_{g}$ so defined need not be n.c. at $g$ ! For example, let $X=[0, \infty), g(x)=1 /(x+1)$, and $f(x)=x / 2$. Then $h(x)=2 x+1$ satisfies $f(g(x))=g(h(x))$ for $x \in[0, \infty)$. However, there exists no $k \in H_{g}$ such that $h(g(x))=g(k(x))$; that is, $2(x+1)^{-1}+1=(k(x)+1)^{-1}$ (note that $x, k(x) \geq 0$ ).

Note that the map $g(x)=1 /(x+1)$ was not surjective. So consider the following example. 
EXAMPLE 2.5. Let $X$ be any set and let $g: X \rightarrow X$ be surjective. Then the family of all self mappings of $X, \mathscr{F}=\{f \mid f: X \rightarrow X\}$, is n.c. at $g$. For suppose $f \in \mathscr{F}$; we need $h \in \mathscr{F}$ such that $f g(x)=g h(x)$ for all $x \in X$. So let $a \in X$. Since $g$ is onto, we can choose $x_{a} \in X$ such that $g\left(x_{a}\right)=f(g(a))$. Choose such an $x_{a}$ for each $a \in X$ and define $h(a)=x_{a}$. Then $h: X \rightarrow X$ and $g(h(a))=g\left(x_{a}\right)=f(g(a))$ for $a \in X$; that is, $f g=g h$.

Proposition 2.6. Suppose that $H$ is a semigroup of maps which is n.c. at $g: X \rightarrow X$. If $f \in H$ and $n \in \mathbb{N}$, there exists $h_{n} \in H$ such that $f g^{n}=g^{n} h_{n}$ (i.e., $H$ is n.c. at $g^{n}$ ).

Proof. Let $f \in H$. Since, $H$ is n.c. at $g$, there exists $h_{1} \in H$ such that $f g=g h_{1}$. So suppose that $k \in \mathbb{N}$ such that $f g^{k}=g^{k} h_{k}$ for some $h_{k} \in H$. Then

$$
f g^{k+1}=\left(f g^{k}\right) g=\left(g^{k} h_{k}\right) g=g^{k}\left(h_{k} g\right) .
$$

Since $h_{k} \in H$, there exists $h_{k+1} \in H$ such that $h_{k} g=g h_{k+1}$, and therefore (2.2) implies $f g^{k+1}=g^{k}\left(g h_{k+1}\right)=g^{k+1} h_{k+1}$, as desired.

Throughout this paper, $P$ denotes a function $P:[0, \infty) \rightarrow[0, \infty)$ which is nondecreasing, and satisfies $\lim _{n \rightarrow \infty} P^{n}(t)=0$ for $t \in[0, \infty)$. (For example, we could let $P(t)=\alpha t$ for some $\alpha \in(0,1)$, or $t /(t+1)$.) And throughout this paper, we appeal to the following lemma.

LEMMA 2.7. Let $H$ be a semi-group of self maps of a set $X$ and suppose that $H$ is nearly commutative at $g: X \rightarrow X$. Let $d: X \times X \rightarrow[0, \infty)$. Suppose that for each pair $x, y \in X$ there exists a choice $r=r(\{x, y\}), s=s(\{x, y\}) \in H$, and $u, v \in\{x, y\}$ for which

$$
d(g x, g y) \leq P(d(r u, s v)) .
$$

Then, if $n \in \mathbb{N}$, for each pair $x, y \in X$ there exist $r_{n}, s_{n} \in H$ and $u_{n}, v_{n} \in\{x, y\}$ such that

$$
d\left(g^{n} x, g^{n} y\right) \leq P^{n}\left(d\left(r_{n} u_{n}, s_{n} v_{n}\right)\right) .
$$

Proof. By (2.3), inequality (2.4) holds for $n=1$, so suppose that $n \in \mathbb{N}$ for which (2.4) is true. Then, if $x, y \in X$,

$$
d\left(g^{n+1} x, g^{n+1} y\right)=d\left(g\left(g^{n} x\right), g\left(g^{n} y\right)\right) \leq P(d(r u, s v)),
$$

where $r, s \in H$ and $u, v \in\left\{g^{n} x, g^{n} y\right\}$, by (2.3). Specifically, $u=g^{n} c, v=g^{n} d$, where $c, d \in\{x, y\}$. And since $r, s \in H$, there exist $r^{\prime}, s^{\prime} \in H$ such that $r g^{n}=g^{n} r^{\prime}$ and $s g^{n}=g^{n} s^{\prime}$, by Proposition 2.6. So (2.4) implies that

$$
d(r u, s v)=d\left(r g^{n}(c), s g^{n}(d)\right)=d\left(g^{n}\left(r^{\prime} c\right), g^{n}\left(s^{\prime} d\right)\right) \leq P^{n}\left(d\left(r_{n} u_{n}, s_{n} v_{v}\right)\right),
$$

where $r_{n}, s_{n} \in H$ and $u_{n}, v_{n} \in\left\{r^{\prime} c, s^{\prime} d\right\}$. Thus, $r_{n} u_{n} \in\left\{\left(r_{n} r^{\prime}\right) c,\left(r_{n} s^{\prime}\right) d\right\}$, where $r_{n} r^{\prime}$ and $r_{n} s^{\prime}$ are elements of $H$, since $H$ is a semi-group. So $r_{n} u_{n}=r_{n+1} u_{n+1}$, where $r_{n+1} \in$ $\left\{r_{n} r^{\prime}, r_{n} s^{\prime}\right\}$ (i.e., $\left.r_{n+1} \in H\right)$ and $u_{n+1} \in\{c, d\} \subset\{x, y\}$. Similarly, $s_{n} v_{n}=s_{n+1} v_{n+1}$, where $s_{n+1} \in H$ and $v_{n+1} \in\{x, y\}$. Thus, (2.6) implies that

$$
d(r u, s v) \leq P^{n}\left(d\left(r_{n+1} u_{n+1}, s_{n+1} v_{n+1}\right)\right), \quad r_{n+1}, s_{n+1} \in H, u_{n+1}, v_{n+1} \in\{x, y\} .
$$


But $P$ is nondecreasing, and therefore (2.7) and (2.5) yield

$$
\begin{aligned}
d\left(g^{n+1} x, g^{n+1} y\right) & \leq P\left(P^{n}\left(d\left(r_{n+1} u_{n+1}, s_{n+1} v_{n+1}\right)\right)\right) \\
& =P^{n+1}\left(d\left(r_{n+1} u_{n+1}, s_{n+1} v_{n+1}\right)\right),
\end{aligned}
$$

with $r_{n+1}, s_{n+1} \in H$ and $u_{n+1}, v_{n+1} \in\{x, y\}$. So, (2.4) is true for all $n$ by induction.

\section{Fixed point theorems}

DEFINITION 3.1. Let $(X ; d)$ be a semi-metric space and let $H$ be a semi-group of self maps of $X$. A map $g: X \rightarrow X$ is $P$-contractive relative to $H$ if and only if (2.3) holds. (We will also say, " $g$ is a P-contraction relative to $H . ")$

LEMMA 3.2. Let $(X ; d)$ be a $T_{2}$ semi-metric space and let $H$ be a semi-group of self maps of $X$ n.c. at $g: X \rightarrow X$. Suppose that $g$ is $P$-contractive relative to $H$ and that $M \subset X$ such that $B=\cup\{H(c) \mid c \in M\}$ is bounded. Then $d\left(g^{n}(x), g^{n}(y)\right) \rightarrow 0$ uniformly on $B$ as $n \rightarrow \infty$. Specifically, if $\epsilon>0$, there exists $k \in \mathbb{N}$ such that

$$
(n \geq k) \Longrightarrow\left(d\left(g^{n}(x), g^{n}(y)\right)<\epsilon \forall x, y \in B\right) .
$$

Proof. By hypothesis $\delta(B)<\infty, P^{n}(\delta(B)) \rightarrow 0$ as $n \rightarrow \infty$. Let $\epsilon>0$. We can choose $k \in \mathbb{N}$ such that

$$
P^{n}(\delta(B))<\epsilon \text { for } n \geq k .
$$

Let $x, y \in B$. If $n \in \mathbb{N}$, since $g$ is $P$-contractive relative to $H$, Lemma 2.7 yields $r_{n}, s_{n} \in H$ and $u_{n}, v_{n} \in\{x, y\}(\subset B)$ such that

$$
d\left(g^{n}(x), g^{n}(y)\right) \leq P^{n}\left(d\left(r_{n} u_{n}, s_{n} v_{n}\right)\right) .
$$

Since $u_{n} \in B$, there exist $h \in H$ and $c \in M$ such that $u_{n}=h(c)$. But $r_{n}, h \in H$, so $r_{n} h \in H$. Therefore, $r_{n} u_{n}=\left(r_{n} h\right)(c) \in H(c) \subset B$. Likewise, $s_{n} v_{n} \in B$. But then $d\left(r_{n} u_{n}, s_{n} v_{n}\right) \leq \delta(B)$ and therefore,

$$
P^{n}\left(d\left(r_{n} u_{n}, s_{n} v_{n}\right)\right) \leq P^{n}(\delta(B)) \text { for } n \in \mathbb{N},
$$

since $P$ is nondecreasing and $n$ is arbitrary. Formulae (3.2), (3.3), and (3.4) imply

$$
d\left(g^{n}(x), g^{n}(y)\right)<\epsilon \text { for } n \geq k .
$$

Since the choice of $k$ in (3.2) was independent of $x$ and $y$, (3.5) holds for all $x, y \in B$.

THEOREM 3.3. Let $(X ; d)$ be a $T_{2}$ semi-metric space, and let $H$ be a semi-group of self maps of $X$ which is n.c. at $g \in H$. Suppose that $H(a)$ is bounded for some $a \in X$ and $X$ is $g$-orbitally complete. If $g$ is a $P$-contraction relative to $H$, then $g^{n}(a) \rightarrow c$ for some $c \in X$. If $g$ is continuous at $c, g(c)=c$.

Proof. Since $X$ is $g$-orbitally complete, to show that $g^{n}(a) \rightarrow c$ for some $c \in X$ it suffices to show that $\left\{g^{n}(a)\right\}$ is a Cauchy sequence. 
To this end, let $\epsilon>0$. Since, $H(a)$ is bounded, Lemma 3.2 with $B=H(a)$ implies that there exists $k \in \mathbb{N}$ such that

$$
n \geq k \Longrightarrow d\left(g^{n}(x), g^{n}(y)\right)<\epsilon \quad \forall x, y \in H(a) .
$$

Therefore, if $m>n \geq k, m=n+r$ for some $r \in \mathbb{N}$, and

$$
d\left(g^{n}(a), g^{m}(a)\right)=d\left(g^{n}(a), g^{n}\left(g^{r}(a)\right)\right)<\epsilon,
$$

since $a, g^{r}(a) \in H(a)$. We conclude that $\left\{g^{n}(a)\right\}$ is Cauchy, and there exists $c \in X$ such that $g^{n}(a) \rightarrow c$.

Now, if $g$ is continuous at $c, \lim _{n \rightarrow \infty} g\left(g^{n}(a)\right)=g(c)$, since $g^{n}(a) \rightarrow c$. But then $g^{n+1}(a) \rightarrow c$ also, so $g(c)=c$ since $(X ; d)$ is a $T_{2}$ semi-metric space.

DefinItion 3.4. Let $X$ and $Y$ be topological spaces. A map $g: X \rightarrow Y$ is closed if and only if $g(M)$ is closed in $Y$ whenever $M$ is a closed subset of $X$.

Note that the conclusion of Lemma 3.2 asserts that $d\left(g^{k}\left(x_{k}\right), g^{k}\left(y_{k}\right)\right) \rightarrow 0$ for any sequences $\left\{x_{k}\right\}$ and $\left\{y_{k}\right\}$ in $B$.

THEOREM 3.5. Let $(X ; d)$ be a bounded and complete $T_{2}$ semi-metric space, and let $H$ be a semi-group of maps n.c. at $g \in H$. If $g$ is closed and $P$-contractive relative to $H$,

(i) there exists $p \in X$ such that $\{p\}=\cap\left\{g^{n}(X) \mid n \in \mathbb{N}\right\}$,

(ii) $p$ is the unique fixed point of $g$,

(iii) $g^{n}(x) \rightarrow p$ for all $x \in X$.

Proof. Let $x \in X$. By Theorem 3.3, $\left\{g^{n}(x)\right\}$ converges to $p$ for some $p \in X$. Moreover, $p \in \cap\left\{g^{n}(X) \mid n \in \mathbb{N}\right\}$. Otherwise, there exists $k \in \mathbb{N}$ such that $p \notin g^{k}(X)$. Since $g^{k}(X)$ is closed, there exists $\epsilon>0$ such that $S(p, \epsilon) \cap g^{k}(X)=\varnothing$. Thus, $d\left(g^{n}(x), p\right) \geq \epsilon$ for $n \geq k$ since $g^{n}(X)$ is a subset of $g^{k}(X)$ for $n \geq k$. This contradicts the fact that $g^{n}(x) \rightarrow p$.

In fact, $\{p\}=\cap\left\{g^{n}(X) \mid n \in \mathbb{N}\right\}$. For if $q \in \cap\left\{g^{n}(X) \mid n \in \mathbb{N}\right\}$, for each $k \in \mathbb{N}$ we can choose $x_{k}, y_{k} \in X$ such that $g^{k}\left(x_{k}\right)=p$ and $g^{k}\left(y_{k}\right)=q$. So

$$
d(p, q)=d\left(g^{k}\left(x_{k}\right), g^{k}\left(y_{k}\right)\right) \longrightarrow 0,
$$

by Lemma 3.2 with $M=X$.

Clearly, (i) implies that $p$ is a fixed point of $g$, since $g(\{p\}) \subset\{p\}$. Thus, if $x \in X$, $d\left(g^{n}(x), p\right)=d\left(g^{n}(x), g^{n}(p)\right) \rightarrow 0$ as $n \rightarrow \infty$, so (iii) holds. Similarly, if $q$ is a fixed point of $g$, then $d(p, q)=\left(g^{n}(p), g^{n}(q)\right) \rightarrow 0$, so that $q=p$. Thus, $p$ is the only fixed point of $g$.

In the following we need the triangle inequality, so we require the underlying space to be a metric space.

THEOREM 3.6. Let $(X, d)$ be a metric space and let $H$ be a semi-group of self maps of $X$ n.c. at some $g \in H$. Suppose that $X$ is $g$-orbitally complete and there exists $k \in \mathbb{N}$ such that for each pair $x, y \in X$, there exist $r, s \in H$ and $u, v \in\{x, y\}$ for which

$$
d\left(g^{k} x, g^{k} y\right) \leq P(d(r u, s v)) .
$$


(i) If there exists $a \in X$ such that $H(a)$ is bounded, then there exists $c \in X$ such that $\lim _{n \rightarrow \infty} g^{n}(a)=c$. If $h$ is continuous for some $h \in H$, then $h(c)=c$. (Specifically, $g(c)=c$ if $g$ is continuous at $c$.)

(ii) If $H(x)$ is bounded for each $x \in X$, there exists a unique $c \in X$ such that $g^{n}(x) \rightarrow c$ for all $x \in X$. If $g$ is continuous at $c, c$ is a unique common fixed point for all $h \in H$.

Proof. Suppose that $H(a)$ is bounded. Since $H$ is n.c. at $g$, Proposition 2.6 says that $H$ is n.c. at $g^{k}$. And $X$ is $g^{k}$-orbitally complete since $X$ is $g$-orbitally complete. Therefore, (3.9) and Theorem 3.3 imply that

$$
\lim _{m \rightarrow \infty}\left(g^{k}\right)^{m}(a)=c \text { for some } c \in X \text {. }
$$

To see that $\lim _{n \rightarrow \infty} g^{n}(a)=c$, let $\epsilon>0$. Then (3.10) and Lemma 3.2 (with $B=H(a)$ ) imply that there exists $p \in \mathbb{N}$ such that $d\left(\left(g^{k}\right)^{p}(a), c\right)<\epsilon / 2$ and $d\left(g^{k p}(x), g^{k p}(y)\right)<$ $\epsilon / 2$ for $x, y \in B$; that is,

$$
d\left(g^{k p}(a), c\right)<\frac{\epsilon}{2}, \quad d\left(g^{k p}\left(g^{i}(a)\right), g^{k p}(a)\right)<\frac{\epsilon}{2} \quad \forall i \in \mathbb{N},
$$

since $g \in H \Rightarrow g^{i}(a) \in H(a)$. So, if $n>k p, n=k p+i$ for some $i \in \mathbb{N}$, and

$$
d\left(g^{n}(a), c\right) \leq d\left(g^{n}(a), g^{k p}(a)\right)+d\left(g^{k p}(a), c\right),
$$

or

$$
d\left(g^{n}(a), c\right) \leq d\left(g^{k p}\left(g^{i}(a)\right), g^{k p}(a)\right)+d\left(g^{k p}(a), c\right)<\frac{\epsilon}{2}+\frac{\epsilon}{2}=\epsilon,
$$

by (3.11). Consequently, $g^{n}(a) \rightarrow c$.

Now, let $h \in H$ and suppose that $h$ is continuous at $c$. Then, $\lim _{n \rightarrow \infty} h\left(g^{n}(a)\right)=h(c)$ and

$$
d(h(c), c)=\lim _{n \rightarrow \infty} d\left(h g^{n}(a), g^{n}(a)\right)=\lim _{n \rightarrow \infty} d\left(h\left(g^{k}\right)^{n}(a),\left(g^{k}\right)^{n}(a)\right) .
$$

But $H$ is n.c. at $g^{k}$, so for $n \in \mathbb{N}$ there exists $h_{n} \in H$ such that $h g^{k n}=g^{k n} h_{n}$. Then, by (3.14),

$$
d(h(c), c)=\lim _{n \rightarrow \infty} d\left(\left(g^{k}\right)^{n}\left(h_{n}(a)\right),\left(g^{k}\right)^{n}(a)\right)=0,
$$

since $a, h_{n}(a) \in H(a)$ and Lemma 3.2 holds for $g^{k}$. Thus, (i) holds.

To prove (ii), suppose that $H(x)$ is bounded for each $x \in X$. If $a, b \in X, g^{n}(a) \rightarrow c_{a}$ and $g^{n}(b) \rightarrow c_{b}$ for some $c_{a}, c_{b} \in X$ by (i). But $c_{a}=c_{b}$, since $H(a) \cup H(b)$ is bounded, and therefore, Lemma 3.2 applied to $g^{k}$ implies that $d\left(c_{a}, c_{b}\right)=\lim _{n \rightarrow \infty} d\left(\left(g^{k}\right)^{n}(a)\right.$, $\left.\left(g^{k}\right)^{n}(b)\right)=0$.

Thus, there exists a unique $c \in X$ such that $g^{n}(x) \rightarrow c$ for all $x \in X$. We know that $g(c)=c$ by part (i), if $g$ is continuous at $c$. Since $g^{n}(d)=d$ for all $n$ if $d$ is a fixed point of $g$, and therefore $g^{n}(d) \rightarrow d$, $c$ must be the only fixed point of $g$. Moreover, $h(c)=c$ for all $h \in H$ (even though $h$ may not be continuous). This follows, since Proposition 2.6 applied to $g^{k}$ implies that for each $n \in \mathbb{N}$,

$$
d(c, h(c))=d\left(\left(g^{k}\right)^{n}(c), h\left(g^{k}\right)^{n}(c)\right)=d\left(\left(g^{k}\right)^{n}(c),\left(g^{k}\right)^{n}\left(h_{n}(c)\right)\right)
$$

for some $h_{n} \in H$. But $H(c)$ is bounded, so Lemma 3.2 applied to $g^{k}$ implies that the right member of (3.16) converges to zero as $n \rightarrow \infty$, and thus, $c=h(c)$. 
REMARK 3.7. Theorem 3.3 appreciably generalizes Theorem 2.1 in [5] and Theorem 3.6 generalizes Corollary 2.3 in [5]-and hence Theorem 2 in [3] and the theorems of Rhoades and Watson [9]. Note that in Theorem 3.6(ii), the mappings $h \in H(h \neq g)$ need not be continuous. Remember also that $C_{g}$ and $O_{g}$ are special instances of $H$.

The following example suggests that the requirement in Theorem 3.6(ii), that $H(x)$ be bounded for each $x \in X$, is not as restrictive as may first appear.

EXAMPLE 3.8. Let $S=\{$ continuous functions $f:[0, \infty) \rightarrow[0, \infty) \mid$ there exists $a_{f} \in(0, \infty)$ such that $f(x)<x$ for $\left.x>a_{f}\right\}$. (So, e.g., $\{f \mid f(x)=m x+b, m \in[0,1$ ) and $b \geq 0\} \subset S$, and $\ln (x+b) \in S$ for $b \geq 1$.) Then (1) $S \cup\left\{i_{d}\right\}$ is a semi-group under composition of functions, and (2) $O_{f}(x)$ is bounded for $f \in S$ and $x \in[0, \infty)$.

First note that, we can let $M_{f}$ denote the maximum value of $f$ on $\left[0, a_{f}\right]$ for each $f \in S$ since each $f$ is continuous. To see that (1) is true, let $f, g \in S$. We need only to show that $g \circ f=g f \in S$. Clearly, $g f$ is a continuous self map of $[0, \infty)$. So let $a_{g f}=\max \left\{a_{f}, M_{g}\right\}$ and suppose that $x>a_{g f}$. We want $g f(x)<x$. Now, $x>a_{g f}$ implies that $x>a_{f}$ so that (i) $f(x)<x$. If $f(x)>a_{g}$, then $g(f(x))<f(x)<x$ by (i) and the definition of $a_{g}$. If $f(x) \leq a_{g}, g(f(x)) \leq M_{g} \leq a_{g f}<x$. So, in any event, $(g \circ f)(x)<x$ if $x>a_{g f}$, and thus, $g \circ f \in S$. (2) follows easily by using induction to show that $\left(f \in S\right.$ ) implies that (if $x \in[0, \infty), f^{n}(x) \leq \max \left\{x, M_{f}\right\}$ for $n \in \mathbb{N}$ ). We omit the details.

If we let $P(t)=\alpha t$ for fixed $\alpha \in(0,1)$ and $t \in[0, \infty)$, we have the following corollary.

COROLLARY 3.9. Let $(X, d)$ be a bounded complete metric space and let $g: X \rightarrow X$ be continuous. Suppose that $H$ is a semi-group of self maps of $X$ n.c. at $g$ and $g \in H$. If there exists $\alpha \in(0,1)$ such that for any pair $x, y \in X$ there exist $r, s \in H$ and $u, v \in\{x, y\}$ for which

$$
d(g x, g y) \leq \alpha d(r u, s v),
$$

then there exists a unique $c \in X$ such that $g^{n}(x) \rightarrow c$ for $x \in X$, and $c=g c=h c$ for all $h \in H$.

\section{Some consequences}

DEFinITION 4.1. A gauge function is an upper semicontinuous (u.s.c.) function $\phi:[0, \infty) \rightarrow[0, \infty)$ such that $\phi(0)=0$ and $\phi(t)<t$ for all $t>0$.

LEMMA 4.2. Let $(X, d)$ be a metric space and let $H$ be a semi-group of self maps of $X$ which is n.c. at $g \in H$. Suppose that $H(x, y)=H(x) \cup H(y)$ is bounded for $x, y \in X$ and there exists a gauge function $\phi$ such that

$$
d(g x, g y) \leq \phi(\delta(H(x, y))) \quad \text { for } x, y \in X .
$$

Then, there exists a nondecreasing continuous function $P:[0, \infty) \rightarrow[0, \infty)$ such that $P^{n}(t) \rightarrow 0$ for all $t>0$ and which satisfies the following condition: for any pair $x, y \in X$ there exist $r=r(x, y), s=s(x, y) \in H$, and $u, v \in\{x, y\}$ such that

$$
d(g x, g y) \leq P(d(r u, s v))
$$


Proof. Let $x, y \in X$ and suppose that (4.1) holds. Since, $\phi$ is a gauge function, as is well known [2], there exists a nondecreasing continuous function $P:[0, \infty) \rightarrow[0, \infty)$ such that $P^{n}(t) \rightarrow 0$ for $t \geq 0$, and

$$
\phi(t)<P(t), \quad P(t)<t \quad \forall t \in(0, \infty) .
$$

Since $P$ is continuous, (4.3) implies that for any $t>0$, there exists $\epsilon_{t} \in(0, t)$ such that

$$
t^{\prime} \in\left(t-\epsilon_{t}, t+\epsilon_{t}\right) \Longrightarrow \phi(t)<P\left(t^{\prime}\right) .
$$

And since $H(x, y)$ is bounded, the definition of $\delta$ implies that there exist $r, s \in H$ and $u, v \in\{x, y\}$ such that, with $t=\delta(H(x, y))$,

$$
t=\delta(H(x, y)) \geq d(r u, s v)>\delta(H(x, y))-\epsilon_{t} .
$$

So, with $t^{\prime}=d(r u, s v),(4.4)$ and (4.5) imply that

$$
\phi(\delta(H(x, y)))<P(d(r u, s v)) .
$$

Therefore, (4.1) implies that $d(g x, g y) \leq P(d(r u, s v))$.

The following theorem provides a generalization of Theorem 2.1 in [2].

THEOREM 4.3. Let $(X, d)$ be a complete metric space and let $H$ be a semi-group of self maps of $X$ which is n.c. at some $g \in H$. Suppose that the following conditions are satisfied:

(i) $H(x)$ is bounded for all $x \in X, g$ is continuous,

(ii) there exists a gauge function $\phi$ and $k \in \mathbb{N}$ such that

$$
d\left(g^{k} x, g^{k} y\right) \leq \phi(\delta(H(x, y))) \quad \text { for } x, y \in X .
$$

Then

(a) $H$ has a unique common fixed point $c$ and $g^{n}(x) \rightarrow c$ for $x \in X$.

(b) If for each $h \in H-\left\{i_{d}\right\}$ there exists $k=k_{h} \in \mathbb{N}$ such that (4.7) holds with $g=$ $h$, then

$$
h^{n}(x) \rightarrow c \quad \forall x \in X, h \in H-\left\{i_{d}\right\} .
$$

Proof. Now, (i) implies that $H(x, y)=H(x) \cup H(y)$ is bounded for $x, y \in X$. To see that (a) is true, note that $H$ is n.c. at $g^{k}$ by Proposition 2.6 and substitute $g^{k}$ for $g$ in Lemma 4.2 to conclude that (3.9) holds. Consequently, we can appeal to Theorem 3.6(ii) to obtain a $c \in X$ such that $g^{n}(x) \rightarrow c$ for $x \in X$. And since $g$ is continuous, $c$ is the unique fixed point of $g$ and a fixed point for each $h \in H$. Thus, $c$ is the unique common fixed point of $H$ (remember, $g \in H$ ) and therefore (a) holds.

To prove (b) note that, by part (a), if $h \in H-\left\{i_{d}\right\}, h \neq g, h^{n}(c)=g(c)=c$ for $n \in \mathbb{N}$. But Theorem 3.6 applied to $h$ yields a unique $c_{1} \in X$ such that $h^{n}(x) \rightarrow c_{1}$ for all $x \in X$. Since $h^{n}(c)=c$ for all $n, c_{1}=c$.

REMARK 4.4. Theorem 4.3 generalizes Theorem 2.1 in [2] in the following ways:

(i) The semi-group $H$ is not required to be near-commutative (i.e., n.c. at each $h \in H)$, but n.c. only at $g$, 
(ii) $g$ is the only member of $H$ required to be continuous,

(iii) in (b), (4.7) is required to hold only for $k=k_{h}$, not for all $k \geq k_{h}$.

Theorem 4.3 yields the following corollary, which generalizes the theorem of Ohta and Nikaido [8] by requiring only that the orbits of $f$-but not all of $X$-be bounded.

COROLLARY 4.5. Let $f$ be a continuous self mapping of a metric space $(X, d)$ having bounded orbits $O_{f}(x)$ for all $x \in X$. If there exist $c \in(0,1)$ and $k \in \mathbb{N}$ such that

$$
d\left(f^{k} x, f^{k} y\right) \leq c \delta\left(\left\{f^{i} t \mid t \in\{x, y\}, i \in \mathbb{N} \cup\{0\}\right\}\right)
$$

for all $x, y \in X$, then $f$ has a unique fixed point.

Observe that Lemma 3.2 does not require that $g \in H$, whereas the theorems in Section 3 do. The requirement that $g \in H$ was convenient in the proof, but the following proposition says that it is not necessary when $O_{g}(a)$ is bounded. Moreover, this result is needed for the proof of Theorem 4.7.

Proposition 4.6. If $H$ is a semi-group of self maps n.c. at $g$ and $g \notin H$, then $H_{g}=\left\{g^{n} h \mid n \in \mathbb{N} \cup\{0\}\right.$ and $\left.h \in H\right\}$ is a semi-group which is n.c. at g. Moreover, $g \in H_{g}$ and $H \subset H_{g}$.

Proof. $H_{g}$ is a semi-group. For if $g^{n} h_{1}, g^{m} h_{2} \in H_{g}$, since $H$ is n.c. at $g$, we have $g^{n} h_{1} g^{m} h_{2}=g^{n}\left(h_{1} g^{m}\right) h_{2}=g^{n}\left(g^{m} h_{3}\right) h_{2}=g^{n+m}\left(h_{4}\right)$, where $h_{4}=h_{3} h_{2} \in H$.

$H_{g}$ is n.c. at $g$, since ( $H$ n.c. at $g$ ) implies that there exists $h_{2} \in H$ such that $\left(g^{n} h\right) g=$ $g^{n}(h g)=g^{n}\left(g h_{2}\right)=g\left(g^{n} h_{2}\right)$.

It is clear that if $g: X \rightarrow X$ is a $P$-contraction relative to $H$, then it is certainly a $P$-contraction relative to $H_{g}$ since $H \subset H_{g}$. We use this fact in the proof of Theorem 4.7.

THEOREM 4.7. Let $C$ be a compact subset of a normed linear space $X$ which is starshaped with respect to $q \in C$. Let $T: C \rightarrow C$ be continuous and let $H$ be a semi-group of affine maps $I: C \rightarrow C$ n.c. at $T$ such that $I(q)=q$. If for each pair $x, y \in C$ there exist $I, J \in H$ and $u, v \in\{x, y\}$ for which

$$
\|T x-T y\| \leq\|I u-J v\|,
$$

then there exists $a \in C$ such that $a=T a$ and $a=I a$ for all continuous $I \in H$.

Proof. Choose a sequence $\left\{k_{n}\right\}$ in $(0,1)$ such that $k_{n} \rightarrow 1$, and for each $n \in \mathbb{N}$, let

$$
T_{n}(x)=k_{n} T x+\left(1-k_{n}\right) q .
$$

Since $C$ is star-shaped with respect to $q, T_{n}: C \rightarrow C$ for $n \in \mathbb{N}$. Moreover, if $I \in H$, there exists $J \in H$ such that

$$
\begin{aligned}
I T_{n} x & =I\left(k_{n} T x+\left(1-k_{n}\right) q\right)=k_{n} I(T x)+\left(1-k_{n}\right) I q \\
& =k_{n} T(J x)+\left(1-k_{n}\right) q=T_{n} J x,
\end{aligned}
$$

since $I$ is affine, $H$ is n.c. at $T$, and $I q=q$. Thus, for each $n \in \mathbb{N}, H$ is a semi-group of affine maps which is n.c. at $T_{n}$. Then, by Proposition 4.6, $H_{T_{n}}$ is a semi-group of self maps of $C$ which is n.c. at $T_{n}, T_{n} \in H_{T_{n}}$, and $H \subset H_{T_{n}}$ for $n \in \mathbb{N}$. 
Now fix $n$. By hypothesis, for each pair $x, y \in C$ there exist $I, J \in H\left(\subset H_{T_{n}}\right)$ and $u, v \in\{x, y\}$ such that

$$
\|T x-T y\| \leq\|I u-J v\|,
$$

so

$$
\left\|T_{n} x-T_{n} y\right\| \leq k_{n}\|I u-J v\|,
$$

by (4.11). Therefore, since $T_{n}$ is continuous and $k_{n} \in(0,1)$, Corollary 3.9 applied to $T_{n}$ and $H_{T_{n}}$ ( $C$ compact implies that $C$ is bounded and complete) implies that there exists a unique $x_{n} \in C$ such that

$$
x_{n}=T_{n}\left(x_{n}\right)=I\left(x_{n}\right) \quad \forall I \in H_{T_{n}} .
$$

Thus we have a sequence $\left\{x_{n}\right\}$ in $C$ which satisfies (4.15). Since $C$ is compact, $\left\{x_{n}\right\}$ has a subsequence $\left\{x_{i_{n}}\right\}$ which converges to some $a \in C$. Equations (4.11) and (4.15) thus imply that

$$
a=\lim _{n \rightarrow \infty} x_{i_{n}}=\lim _{n \rightarrow \infty} k_{i_{n}} T x_{i_{n}}+\lim _{n \rightarrow \infty}\left(1-k_{i_{n}}\right) q=\lim _{n \rightarrow \infty} I x_{i_{n}} .
$$

But $T$ is continuous, so (4.16) implies that $a=T a$, and $a=I a$ for all continuous $I$.

REMARK 4.8. We see that Theorem 4.7 does indeed extend Theorem 3 in [7] if we observe that the family $\mathscr{F}$ in Theorem 3 [7]. is a family of sets which is a subset of $C_{g}$. We can let

$$
H=\left\{\text { maps } h: C \rightarrow C \mid h \text { is affine, } h \in C_{g}\right\} .
$$

Then $H$ is a semi-group and $\mathscr{F} \subset H$.

5. Conclusion. We conclude with further evidence of the generality and applicability of the concept of being nearly commutative at a function $g$. The theorem below generalizes Theorem 4.2 in [5] by replacing the semi-group $C_{g f}$ with a more general semi-group $H$.

THEOREM 5.1. Let $f$ and $g$ be commuting self maps of a compact metric space $(X, d)$ such that $g f$ is continuous. If $H$ is a semi-group of self maps of $X$ which is n.c. at $g f$, and

$$
f x \neq g y \Longrightarrow d(f x, g y)<\delta(H(x, y)),
$$

then there exists a unique point $a \in X$ such that $a=f a=g a=h a$ for all $h \in H$.

We leave the proof of Theorem 5.1 to the interested reader.

\section{REFERENCES}

[1] T. L. Hicks and B. E. Rhoades, Fixed points for pairs of mappings in d-complete topological spaces, Int. J. Math. Math. Sci. 16 (1993), no. 2, 259-266. MR 93k:54076. Zbl 796.54049.

[2] Y.-Y. Huang and C.-C. Hong, Common fixed point theorems for semigroups on metric spaces, Int. J. Math. Math. Sci. 22 (1999), no. 2, 377-386. MR 2000b:54053. Zbl 944.47034.

[3] J. Jachymski, A generalization of the theorem by Rhoades and Watson for contractive type mappings, Math. Japon. 38 (1993), no. 6, 1095-1102. MR 94k:54076. Zbl 795.54054. 
[4] J. Jachymski, J. Matkowski, and T. Świạtkowski, Nonlinear contractions on semimetric spaces, J. Appl. Anal. 1 (1995), no. 2, 125-134. CMP 1395268.

[5] G. Jungck, Common fixed points for commuting and compatible maps on compacta, Proc. Amer. Math. Soc. 103 (1988), no. 3, 977-983. MR 89h:54030. Zbl 661.54043.

[6] _ Fixed point theorems for semi-groups of self maps of semi-metric spaces, Int. J. Math. Math. Sci. 21 (1998), no. 1, 125-132. CMP 1486 967. Zbl 892.54026.

[7] G. Jungck and S. Sessa, Fixed point theorems in best approximation theory, Math. Japon. 42 (1995), no. 2, 249-252. MR 96f:47112. Zbl 834.54026.

[8] M. Ohta and G. Nikaido, Remarks on fixed point theorems in complete metric spaces, Math. Japon. 39 (1994), no. 2, 287-290. MR 94m:54099. Zbl 802.47055.

[9] B. E. Rhoades and B. Watson, Generalized contractions and fixed points in metric spaces, Math. Japon. 34 (1989), no. 6, 975-982. MR 91c:54057. Zbl 697.54023.

Gerald F. Jungck: Department of MAthematics, Bradley University, PeORIA, IL 61625, USA

E-mail address: gfj@hi17top.brad1ey.edu 


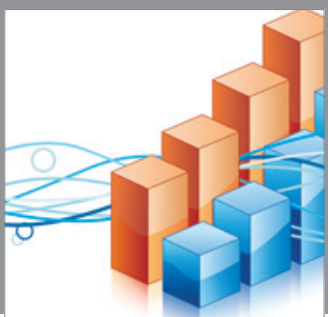

Advances in

Operations Research

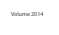

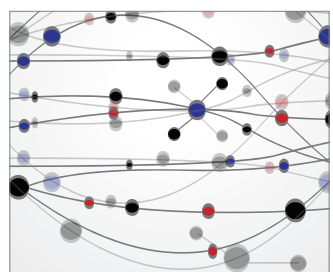

\section{The Scientific} World Journal
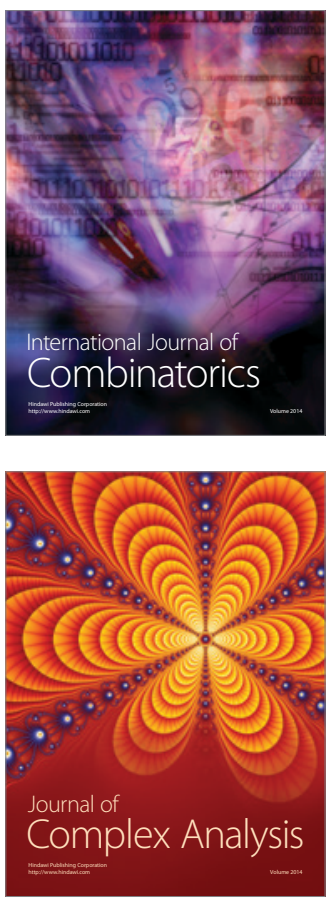

International Journal of

Mathematics and

Mathematical

Sciences
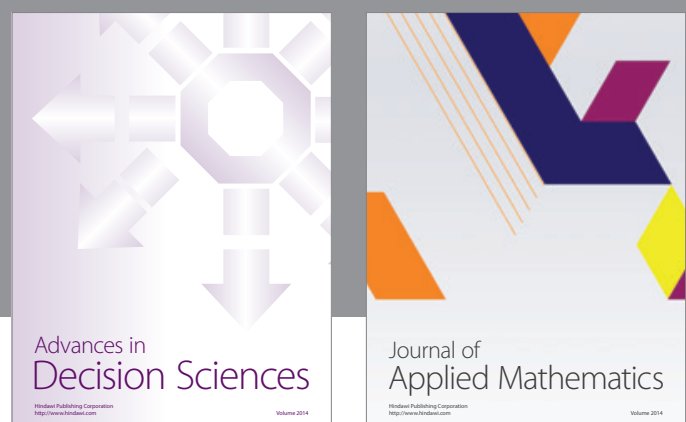

Journal of

Applied Mathematics
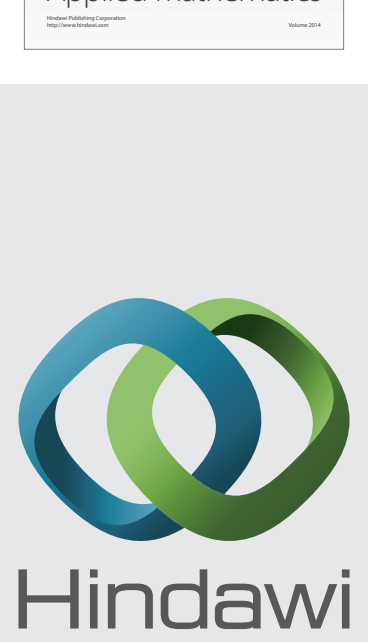

Submit your manuscripts at http://www.hindawi.com
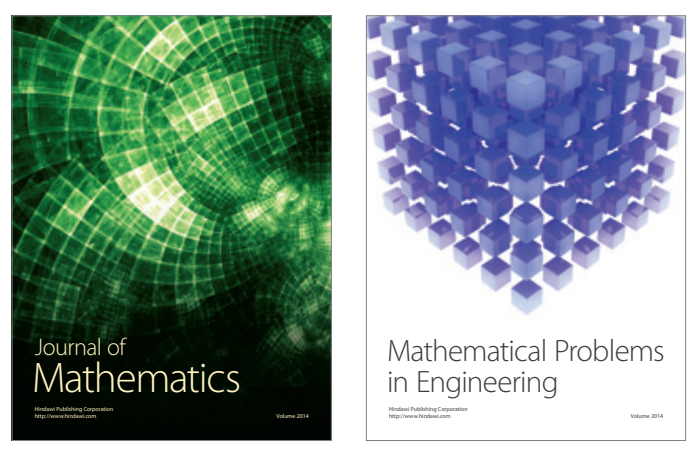

Mathematical Problems in Engineering
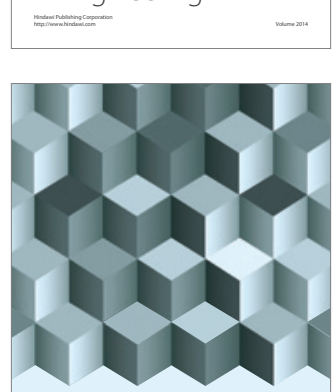

Journal of

Function Spaces
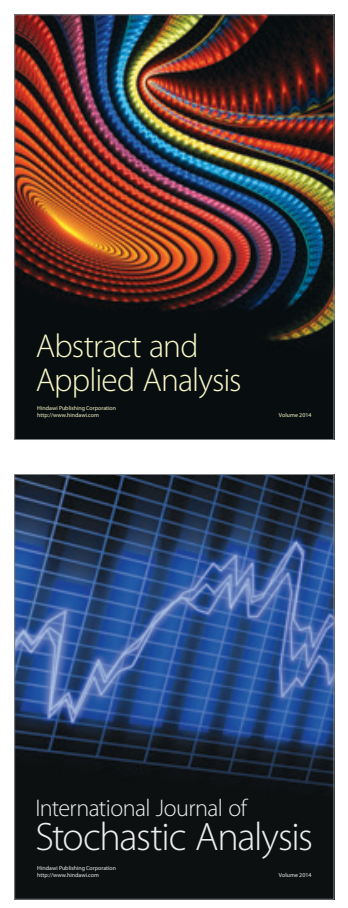

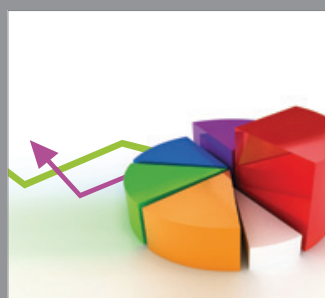

ournal of

Probability and Statistics

Promensencen
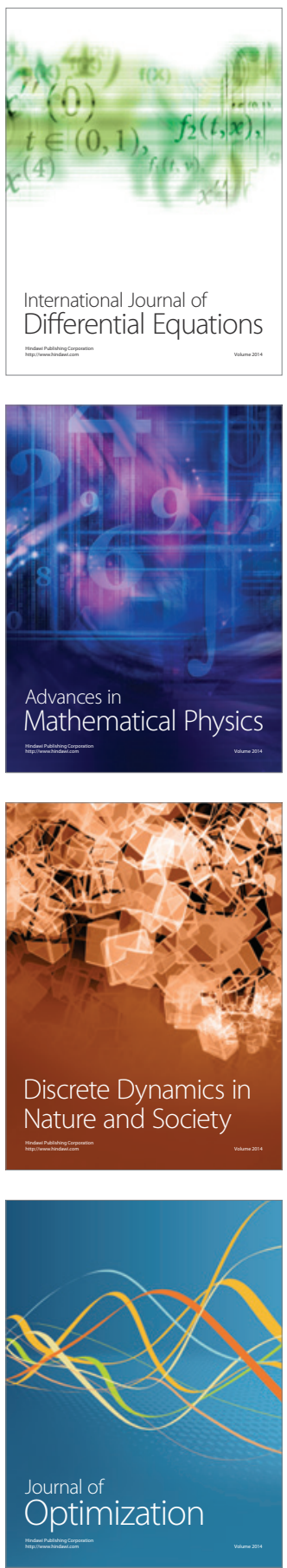